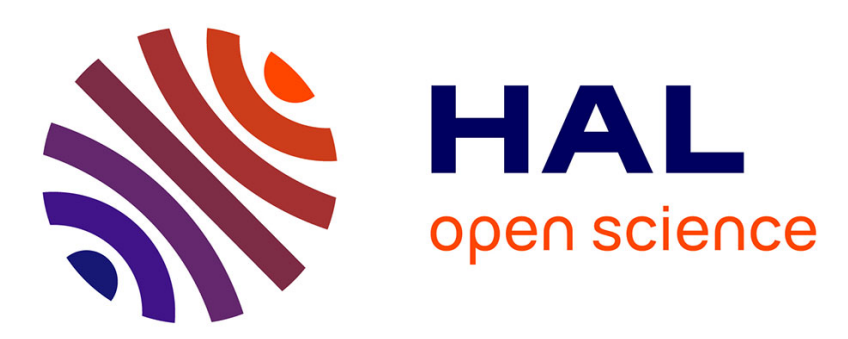

\title{
d-Wave Superconductivity in the Large-Degeneracy Limit of the Anderson Lattice
}

M. Lavagna, A. J. Millis, P.A. Lee

\section{To cite this version:}

M. Lavagna, A. J. Millis, P.A. Lee. d-Wave Superconductivity in the Large-Degeneracy Limit of the Anderson Lattice. Physical Review Letters, 1987, 58 (3), pp.266 - 269. 10.1103/PhysRevLett.58.266 . hal-01896327

\section{HAL Id: hal-01896327 https://hal.science/hal-01896327}

Submitted on 16 Oct 2018

HAL is a multi-disciplinary open access archive for the deposit and dissemination of scientific research documents, whether they are published or not. The documents may come from teaching and research institutions in France or abroad, or from public or private research centers.
L'archive ouverte pluridisciplinaire HAL, est destinée au dépôt et à la diffusion de documents scientifiques de niveau recherche, publiés ou non, émanant des établissements d'enseignement et de recherche français ou étrangers, des laboratoires publics ou privés. 


\title{
$d$-Wave Superconductivity in the Large-Degeneracy Limit of the Anderson Lattice
}

\author{
M. Lavagna, (a) A. J. Millis, ${ }^{(b)}$ and P. A. Lee \\ Department of Physics, Massachusetts Institute of Technology, Cambridge, Massachusetts 02139 \\ (Received 10 June 1986)
}

\begin{abstract}
We show, within a Kondo-boson $1 / N$ expansion, that the infinite- $U$ lattice Anderson model is unstable to $d$-wave superconductivity in the limit in which the model describes heavy-fermion behavior. The pairing mechanism is Kondo-boson exchange between heavy quasiparticles found in mean-field theory. The model is extended to include the Coulomb pseudopotential.
\end{abstract}

PACS numbers: $74.20 .-\mathrm{z}, 72.10 .-\mathrm{Qm}, 75.20 . \mathrm{Hr}$

The heavy-fermion superconductors have been of considerable and continuing interest to condensed-matter physicists since their discovery seven years ago. ${ }^{1}$ It is now believed that the superconducting state in the heavy-fermion materials is "unconventional" in two senses. One sense has to do with the form of the gap function, which appears to vanish along points or lines on the Fermi surface, ${ }^{2}$ unlike the gap in conventional superconductors, which is essentially isotropic over the Fermi surface. One infers from this that heavy-fermion superconductivity involves electrons paired in a state of nonzero angular momentum, unlike conventional superconductors, in which the electrons form $s$-wave pairs. The other, perhaps more fundamental, unconventionality is that the pairing interaction that causes heavy-fermion superconductivity is unlikely to be the conventional electron-phonon interaction that leads to superconductivity in ordinary metals. ${ }^{3}$

In this paper we start from the $U=\infty$ lattice Anderson model, which is believed ${ }^{3}$ to contain the essential physics of the heavy-fermion materials and which contains only electron-electron interactions. We show within a "slave boson" large-degeneracy expansion, originally devised for the one-impurity Anderson model ${ }^{4,5}$ and recently extended to a lattice, ${ }^{6,7}$ that the model is unstable with respect to $d$-wave superconductivity.

The $U=\infty$ Anderson model describes an essentially structureless band of conduction $(c)$ electrons hybridizing with a localized set of $f$ electrons and subject to the constraint that the number of $f$ electrons on any lattice site $i, n_{f} \leq 1$. To obtain a tractable theory we have assumed both $c$ and $f$ electrons have "spin $N$." In the slave-boson technique, one introduces a new Bose field, $b_{i}^{\dagger}$, which creates a hole on the $f$ orbital on site $i$, replaces the constraint $n_{f}^{i} \leq 1$ by

$$
Q_{i}=n_{f}^{i}+n \dot{b}=1,
$$

and writes the Hamiltonian

$$
H=\sum_{k, m} \varepsilon_{k} c_{k m}^{\dagger} c_{k m}+E_{0} f_{k m}^{\dagger} f_{m k}+\left(\frac{V}{\sqrt{N}} \sum_{k, q, m} c_{k m}^{\dagger} f_{k+q m} b_{q}^{\dagger}+\text { H.c. }\right)
$$

All energies are measured from the chemical potential, $\mu$. We are interested in the Kondo limit, in which $\eta=-E_{0} / \rho_{0} V^{2}>1$. $\rho_{0}$ is the $c$-electron density of states. The model given by Eqs. 1(a) and 1(b) is equivalent to the original Anderson model, but may be studied with use of conventional field-theoretical techniques. We note that the $f$ operator in Eq. (1) does not have a simple physical meaning; the creation operator for a physical $f$ state is given ${ }^{4}$ by $\left(f^{\dagger} b\right)$.

One studies the low-temperature properties of the system (1) by adding to Eq. 1(b) a Lagrange multiplier field $\lambda$ to enforce the constraint ( $1 \mathrm{a}$ ), and then decomposing $b$ and $\lambda$ into mean-field and fluctuating components. If one retains only the mean-field parts of $b$ and $\lambda$, the model may easily be solved. This mean-field theory is exact as $N \rightarrow \infty^{4,5}$ Corrections to the mean- field theory are due to interactions between the fermions and the fluctuating parts of the Bose operators; these corrections may be expanded in powers of $1 / N$. The problem may be formulated in either the Cartesian or the radial gauge. In the former we write $b$ $=\sqrt{N}(a+A)$ and consider $A$ as the fluctuating field. In the Cartesian formulation, infrared divergences appear at intermediate stages of many calculations and must be carefully canceled from physical results. ${ }^{6,8}$ It is often more convenient to use the radial gauge ${ }^{5,7}$ in which one rewrites the problem in terms of new Bose fields $r_{i}$ and $\theta_{i}$ defined via $b_{i}^{\dagger}=r_{i} e^{i \theta_{i}}$ and a new Fermi operator $f_{i}^{\prime}=f_{i} e^{i \theta_{i}}$. Then, following Ref. 5, we may (after some algebraic manipulation) rewrite the problem in functional integral form with a Lagrangean given, as a sum of 
fermion, boson, interaction, and constraint terms, by $L(t)=L_{\mathrm{F}}+L_{\mathrm{B}}+L_{I}+L_{C}$, where

$$
\begin{aligned}
& L_{\mathrm{F}}=\sum_{k, m}\left(c_{k m}^{\dagger} f_{k m}^{\dagger \dagger}\right)\left(\begin{array}{cc}
\partial_{\tau}+\varepsilon_{k} & \sigma_{0} \\
\sigma_{0} & \partial \tau+\varepsilon_{f}
\end{array}\right)\left(\begin{array}{l}
c_{k m} \\
f_{k m}^{\prime}
\end{array}\right), \\
& L_{\mathrm{B}}=\frac{N}{V^{2}} \sum_{k}\left(\begin{array}{ll}
\sigma_{k} & \lambda_{k}
\end{array}\right)\left(\begin{array}{cc}
\varepsilon_{f}-E_{0} & i \sigma_{0} \\
i \sigma_{0} & 0
\end{array}\right)\left(\begin{array}{l}
\sigma_{-k} \\
\lambda-k
\end{array}\right), \\
& L_{I}=\sum_{k, k, m}\left(c_{k m}^{\dagger} f_{k m}^{\prime \dagger}\right)\left(\begin{array}{cc}
0 & \sigma_{k-k^{\prime}} \\
\sigma_{k}-k^{\prime} & i \lambda_{k-k^{\prime}}
\end{array}\right)\left(\begin{array}{l}
c_{k^{\prime} m} \\
f_{k^{\prime} m}^{\prime}
\end{array}\right)+\frac{N}{V^{2}} \sum_{k, k^{\prime}} i \lambda_{k} \sigma-k^{\prime} \sigma_{k^{\prime}-k}, \\
& L_{C}=N\left(\frac{\sigma_{0}^{2}}{V^{2}}-q_{0}\right)\left(\varepsilon_{f}-E_{0}\right) .
\end{aligned}
$$

Here $\sigma$ and $\lambda$ are real scalar bose fields related to the fluctuating parts of $V r$ and $(\partial \theta / \partial \tau+\lambda) ; \sigma_{0}=V a$ and $\varepsilon_{f}$ are parameters determined by minimizing the free energy and related to the static parts of $V r$ and $(\partial \theta / \partial \tau+\lambda)$. $q_{0}$ is defined as $Q_{i} / N$. The model defined by Eqs. (2) is only equivalent to the original Anderson model if $q_{0}=1 / N$, but to obtain a sensible $1 / N$ expansion we must regard $q_{0}$ as a parameter independent of the explicit $N$ in Eqs. (4), and only set $q_{0}=1 / N$ at the end of a calculation. ${ }^{6}$

The mean-field theory corresponds to electrons moving in the band structure determined by $L_{\mathrm{F}}$. This band structure has a gap centered at $\varepsilon_{f}$. To leading order in $1 / N$ the parameters $\sigma_{0}$ and $\varepsilon_{f}$ are (in the limit $\eta \gg 1$ )

$$
\begin{aligned}
& \varepsilon_{f}=W \exp \left(-\left|E_{0}\right| / \rho_{0} V^{2}\right), \\
& \sigma_{0}^{2}=q_{0} V^{2}\left(1-n_{f}\right)\left(1-n_{f}^{2} \alpha / 4\right)+O\left(q_{0}^{3}\right), \\
& n_{f}=\left(1+\varepsilon_{f} / \rho_{0} V^{2}\right)^{-1} .
\end{aligned}
$$

$W$ is the energy of the bottom of the $c$-electron band, measured from the chemical potential, $\mu . \rho_{0}$ is the $c$ electron density of states at $\varepsilon_{k}=\mu . n_{f}$ is the mean-field, leading order in $q_{0}$, number of $f$ electrons. It is not the same as the physical number of $f$ electrons. ${ }^{9} \alpha$ $=q_{0} / \rho_{0} W$. We assume one has $\sim 1 c$ electrons per spin channel. $\alpha$ is thus $O\left(q_{0}\right) . \varepsilon_{f}$, the smallest energy scale in the theory, is the effective Fermi energy for the lowlying quasiparticle excitations. ${ }^{6} \sigma_{0}$ is the effective hybridization. We have assumed that the Fermi level lies in the lower band; essentially equivalent results are obtained if it lies in the upper one. It is convenient to work with the quasiparticle operators $d_{k 1}$ and $d_{k 2}$ which diagonalize $H_{0}$ and pertain to the lower and upper bands respectively. One finds $d_{k i}=\cos \theta_{k i} c_{k i}+\sin \theta_{k i} f_{k i}^{\prime}$, where $\left(E_{k i}\right.$ is the energy in band $\left.i\right) \tan \theta_{k i}=\left(E_{k i}-\varepsilon_{k}\right) /$ $\sigma_{0}=\sigma_{0} /\left(E_{k i}-\varepsilon_{f}\right)$.

The density of states at the Fermi surface, $\rho$, is very large; the quasiparticles are therefore heavy fermions of mass enhancement $m^{*} / m=\left(\sigma_{0} / \varepsilon_{f}\right)^{2} \gg 1$. The spectral weight of the $c$ electrons at the Fermi surface is $m / m^{*} \ll 1$. The mass enhancement appears in the specific-heat coefficient $\gamma$, which may be written in the usual Fermi-liquid form $\gamma=N k_{\mathrm{B}}^{2} m^{*} k_{\mathrm{F}} / 6$ or, if $\gamma$ is normalized to the number of $f$-electron sites, $\gamma=\pi^{2} k_{\mathrm{B}}^{2} / 3 \varepsilon_{f}$.

Corrections to the mean-field theory are due to electron-boson interactions, which are small because the boson propagator contains a factor of $1 / N$. However, we shall show that the electron-boson interaction leads to $d$-wave superconductivity in the model defined by Eqs. (2). We proceed by investigating divergences in the scattering amplitude, $\Gamma$, for two quasiparticles of opposite momenta near the Fermi surface. We note that the quasiparticle scattering amplitude is conventionally decomposed into spin singlet and triplet channels; this is meaningless in our spin- $N$ model. An alternative procedure is to decompose the scattering amplitude into orbital angular momentum channels only. This is justified because the boson propagator does not carry spin.

We write the series for $\Gamma$ in Fig. $1 ; \Gamma$ is a function only of angle between $k$ and $k^{\prime}, \varphi$. If $q=k^{\prime}-k$, then $|q|$ $=2 k_{\mathrm{F}} \sin \varphi / 2$. We define the interaction in the $l$ th orbital-angular-momentum channel by

$$
\Gamma_{l}=[(2 l+1) / 2] \int d \varphi \sin \varphi P_{l}(\cos \varphi) \Gamma(\varphi) .
$$

As shown in Fig. 1, $\Gamma$ may be written as a ladder sum of particle-particle propagators linking the two-particleirreducible (2PI) vertex $\Gamma_{0}$. To leading order in $1 / N$ it is given by the sum of graphs shown in Fig. 2. The resulting expressions are lengthy due to the matrix nature of the boson propagator, and will be presented in a longer paper. We note that $\Gamma_{0}$ is of order $1 / N$; in fact, that part

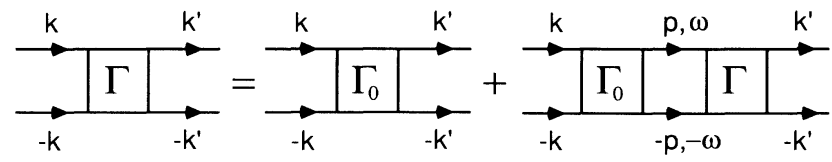

FIG. 1. Expansion for the particle-particle scattering amplitude $\Gamma$ in terms of the two-particle-irreducible (2PI) amplitude $\Gamma_{0}$. The solid lines with arrows are electron Green's functions; they may be in either band. To leading order in $1 / N$ they may be approximated by noninteracting Green's functions. $|k|$ $=\left|k^{\prime}\right|=k_{\mathrm{F}} ; p_{i}$ and $\varepsilon_{i}$ vary over all possible values. 


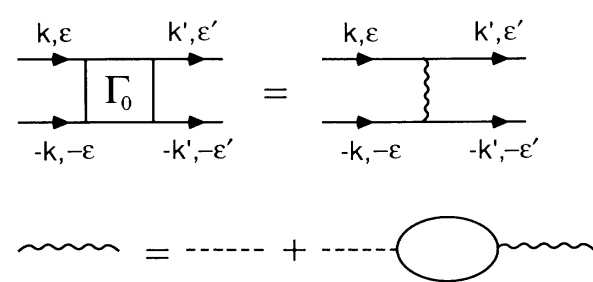

FIG. 2. Expression for the $2 \mathrm{PI}$ amplitude $\Gamma_{0}$. The wiggly line is the full boson propagator. The dashed line represents the matrix of bare-boson propagators, obtained by inverting $L_{\mathrm{B}}$, Eq. 2 (b). The bubble represents the various possible fermion loops that make up the leading-order boson self-energies; they are computed with use of noninteracting Green's functions, obtained from $L_{\mathrm{F}}$ [Eq. $2(\mathrm{a})$ ] and are coupled to the boson propagators via the first term of $L_{I}$ [Eq. 2(c)]. The momentum across the boson line is $q=k^{\prime}-k$.

of $\Gamma_{0}$ which varies with the momentum transfer across the boson line is of order $q_{0} / N$. Thus we need only consider contributions to the full vertex $\Gamma$ in which the smallness of the 2PI vertex is compensated by a logarithmic divergence arising from a particle-particle propagator. We find that logarithmic divergences only occur when both intermediate lines are in the lower band and the energy $\omega$ and momentum $p$ are such that $|\omega|$, $\left|E_{p 1}\right|<\varepsilon_{f}$. In this region the $2 \mathrm{PI}$ vertex $\Gamma_{0}$ is essentially independent of $|\omega|$ and $|p|$ and also of $\varepsilon$, the energy across the boson line. $\Gamma_{0}(q, \omega)$ does have structure at small $q$ and $\omega$ due to a zero-sound collective mode; because of the high velocity of this mode, it contributes only for a negligible region of $q$, and is thus unimportant for the pairing interaction. $s$ in the weak-coupling electron-phonon problem, we may, to logarithmic accuracy, replace the full interaction by its static limit, $\Gamma_{0}(q, \varepsilon=0)$, and cut off the energy integrals at $\varepsilon=\varepsilon_{f}$. We emphasize that unlike the usual electron-phonon problem, the upper cutoff comes from the curvature of the quasiparticle band as well as from the $\omega$-space structure of the ineraction $\Gamma_{0}(q, \omega)$.

After decomposing $\Gamma_{0}$ as in Eq. (4) and projecting onto the $l$ orbital angular momentum channel, we may immediately write

$$
\Gamma_{l}=\Gamma_{0 l}\left[1+\left(\rho \Gamma_{0 l} / 2 l+1\right) \ln \left(\varepsilon_{f} / T\right)\right]^{-1} .
$$

The criterion for $l$-wave superconductivity is $\Gamma_{0 l}<0$.

We have computed $\Gamma_{0}(q, \varepsilon=0)$; assuming $\varepsilon_{k} \sim k^{2}$, and expanding for small $q_{0}$, we find

$$
\Gamma_{0}(q, \varepsilon=0)=\frac{1}{N \rho}\left(1-\left(1-n_{f}\right)^{2}+n_{f}^{2} \alpha\left(1-\frac{4}{3} n_{f}+\frac{1}{2} n_{f}^{2}\right)-\frac{n_{f}^{2} \alpha}{12} f\left(q / 2 k_{\mathrm{F}}\right)\left(3 n_{f}^{2}-9 n_{f}+7\right)\right),
$$

where $f(x)=(1 / x) \ln [|(1+x) /(1-x)|]$.

For the $\Gamma_{0 l}$ we find $\rho \Gamma_{0 s}>0, \quad \rho \Gamma_{0 p}>0$, and $\rho \Gamma_{0 d}=-0.01 \alpha / n_{f}^{2}\left(3 n_{f}^{2}-9 n_{f}+7\right) ;$ the $\Gamma_{0 l}$ are very small for $l>2$. We therefore expect the boson-mediated interaction to give $d$-wave $(l=2)$ superconductivity. We note that the $s$-channel interaction is repulsive because of the hard-core repulsion built into the model by the constraint 1 (a). We also note that in an exchange model (such as the one we consider) $\rho \Gamma_{0 l}=-A q$, where $A q^{f}$ is the antisymmetric Landau parameter. Our results for the landau parameters agree with those of Ref. 7, in the limit $n_{f} \rightarrow 1$.

Note that the quasiparticles involve the $f^{\prime}$ operators, which exist only in the radial gauge, and whose physical meaning is obscure. Note also that $\Gamma$ is infrared divergent if calculated in the Cartesian gauge. Thus, one may wonder about the meaning of any computation of $\Gamma$. A gauge-invariant, physically meaningful, criterion for $L$ wave superconductivity is the divergence of the correlation function

$$
\left\langle\sum_{k} Y_{L m}(k) c_{k m}^{\dagger} c \unlhd_{k m^{\prime}}^{\dagger} \sum_{k^{\prime}} Y_{L m}\left(\hat{\mathbf{k}}^{\prime}\right) c_{k^{\prime} m} c-k^{\prime} m^{\prime}\right\rangle
$$

which involves only $c$ operators and is therefore a physical quantity. We have computed this quantity to leading and next to leading orders in $1 / N$ in both the radial and Cartesian gauge formulations. We obtain a series proportional to $\ln \left(\varepsilon_{f} / T\right)+\lambda \ln ^{2}\left(\varepsilon_{f} / T\right)$. By assuming that the series can be summed to give $\ln \left(\varepsilon_{f} / T\right)[1-\lambda$ $\left.\times \ln \left(\varepsilon_{f} / T\right)\right]^{-1}$ we obtain a criterion for superconductivity which is identical to that obtained from the calculation of $\Gamma$ above. This indicates that the superconducting instability found among quasiparticles defined in the radial gauge corresponds to a physical superconducting instability.

We now extend the Anderson model to include Coulomb interactions involving the $c$ electrons. Two additional terms are possible: We write them $U_{c c} n_{c}(r) n_{c}\left(r^{\prime}\right)$ and $U_{f c} n_{f}(r) n_{c}\left(r^{\prime}\right)$. The $f f$ interaction is already included in the Anderson model. We are interested in the short-range part of the interaction; $U_{c c}$ and $U_{c f}$ are therefore phenomenological Fermi-liquid parameters. We expect these residual interactions to be weak; thus $N \rho_{0} U<1$. (The factor $N$ arises because $\rho_{0}$ is the density of states per spin.) We have shown that the term in $U_{c f}$ is negligible compared to that in $U_{c c}$ by using the constraint (1) to rewrite $n_{f}$ in terms of Bose operators. A straightforward extension of the usual derivation of the Coulomb pseudopotential shows that the effects of $U_{c c}$ may be incorporated by writing a new effective interaction, which in the $l=2$ channel is

$$
\lambda_{\text {eff }}=-\left[\rho \Gamma_{0 d}+\left(m / m^{*}\right) \mu^{*} / N\right] /(2 l+1) \text {. }
$$

Here $\mu^{*}=\mu /\left(1+\mu \ln W / \sigma_{0}\right)$ is the $l=2$ projection of $N \rho_{0} U_{c c}$, rescaled via the Anderson-Morel scaling. The factor $\left(\mathrm{m} / \mathrm{m}^{*}\right)$ comes from the combination of the small 
weight of the $c$ electrons at the Fermi surface and the density-of-states enhancement.

Equation (7) immediately yields

$$
T_{c}=\varepsilon_{f} \exp \left(-1 / \lambda_{\text {eff }}\right) \text {. }
$$

We make the following comments: (i) Although the attractive part of our interaction is of order $q_{0} / N \sim 1 / N^{2}$, our calculation, which included only $1 / N$ contributions to $\Gamma_{0}$, is consistent. This is because the dressed-boson propagator may be written as the sum of two terms: one independent of the momentum $q$ carried by the boson, and one, of order $q_{0}$ relative to the first, which does depend on $q$. Therefore any two boson contributions to $\Gamma_{0}$ would contribute to $\Gamma_{0 l}=0$ in order $q_{0} / N=1 / N^{2}$ but would only contribute to $\Gamma_{0 l \neq 0}$ in order $q_{0} / N^{2}=1 / N^{3}$. (ii) The attractive pairing interaction is specific to the lattice: In the one-impurity version of our model, one finds that the $s$-channel interaction is repulsive and all other interaction amplitudes are zero. (iii) Equation (8) is closely related to the Patton-Zaringhalan (PZ) formula ${ }^{10}$ and its generalization by Levin and Vall. ${ }^{11}$ The PZ formula is a phenomenological expression for $T_{c}$ in terms of Landau parameters under a particular assumption about extrapolating the quasiparticle interaction amplitude away from the forward scattering limit, and with the upper cutoff a parameter to be determined. Levin and Valls ${ }^{11}$ have generalized the PZ formula to the "exchange model" case in which the interaction amplitude depends only on the momentum and frequency exchanged by the interacting particle, and have derived the generalized $\mathrm{PZ}$ formula for the $T_{c}$ of ${ }^{3} \mathrm{He}$ from a microscopic model in which quasiparticles exchange paramagnons. Our model is an exchange model; however, we emphasize that in our approach, as well as that of Levin and Valls, the starting point is a microscopic model which yields an expression for the full frequency-dependent interaction $\Gamma(q, \omega)$ from which the interaction and (up to factors of order unity) the cutoff can be derived. ${ }^{12}$ (iv) As in the usual weak-coupling theory of superconductivity, our calculation is valid to leading logarithmic order; thus we have determined the leading term (in $1 / N$ in $\lambda_{\text {eff }}$ and have determined the cutoff up to factors of order unity. (v) The values obtained for $\rho \Gamma_{0 l}$ are nonuniversal, and depend on details of band structure, of hybridization, etc. (vi) Because the Coulomb term is multiplied by $\mathrm{m} / \mathrm{m}^{*}$, it is negligible in the limit $\eta \gg 1$, and superconductivity is possible despite the weakness of the pairing interaction. As $\eta \rightarrow \infty$, however, Eqs. 3(a), 3(b), and (8) show that the decrease in $\varepsilon_{f}$ drives $T_{c}$ to zero. As $\eta$ decreases towards the intermediate valence regime $\eta \sim 1$, although $\varepsilon_{f}$ increases, $n_{f}$ and $m^{*}$ decrease, and superconductivity is destroyed. Note that the leading corrections to $\lambda_{\text {eff }}$ are proportional to $\left(1-n_{f}\right)^{2}$ for $n_{f} \cong 1$. Thus, as $\eta$ decreases from $\infty T_{c}$ must increase, pass through a maximum at some $\eta=\eta_{\max }$, and then decrease. (vii) It seems possible to vary $\eta$ experimentally by applying pressure $P$. In Ce and $\mathrm{U}$ compounds, $d \eta / d P<0^{2}$. In $\mathrm{CeCu}_{2} \mathrm{Si}_{2}, T_{c}(P)$ has the qualitative behavior expected if $\eta(P=0)>\eta_{\max }$ : It initially increases with pressure, then passes through a maximum and decreases. ${ }^{13,14}$ In $\mathrm{UPt}_{3}{ }^{15}$ and $\mathrm{UBe}_{13},{ }^{16}$ $T_{c}(P)$ monotonically decreases with increasing $P$, as expected if $\eta(P=0)<\eta_{\max }$.

In conclusion, we have shown that in the Anderson lattice there is a nonphonon mechanism which leads to a weak, $d$-wave superconducting instability, at least within our $1 / N$ model. The superconducting instability is specific to the heavy-fermion state, and is due to slaveboson exchange. We note that the slave boson does not carry spin: The superconductivity which we find is not associated with spin fluctuations. Rather, it is due to $f$ density fluctuations subject to the infinite- $U$ constraint.

This research has been supported by National Science Foundation Grant No. DMR-85-21377, and an AT\&T Bell Laboratories Ph.D. Scholarship (for A.J.M.), and an National Science Foundation-Centre National de la Recherche Scientifique (France) Exchange Award (for M.L.). We thank Dr. A. Auerbach for a useful discussion.

(a) On leave from Laboratoire Louis Néel, Centre National de la Recherche Scientifique, 166X 38042 Grenoble Cedex, France.

(b) Current address: AT\&T Bell Laboratories, Murray Hill, New Jersey 07974.

${ }^{1}$ F. Steglich, J. Aarts, C. D. Bredl, W. Lieke, D. Meschede, W. Franz, and J. Shafer, Phys. Rev. Lett. 43, 1892 (1982).

${ }^{2}$ For an experimental review, see G. R. Stewart, Rev. Mod. Phys. 56, 755 (1984).

${ }^{3}$ P. A. Lee, T. M. Rice, J. W. Serene, L. J. Sham, and J. W. Wilkins, Comm. Solid State Phys. 12B, 99 (1986).

${ }^{4}$ P. Coleman, Phys. Rev. B 29, 3035 (1984).

${ }^{5}$ N. Read and D. M. Newns, J. Phys. C 16, 3273 (1983).

${ }^{6}$ A. J. Millis and P. A. Lee, Phys. Rev. B (to be published).

${ }^{7}$ A. Auerbach and K. Levin, Phys. Rev. Lett. 57, 877 (1986).

${ }^{8}$ N. Read, J. Phys. C 10, 2651 (1985).

${ }^{9}$ We thank N. Read (private communication) for drawing our attention to this point.

${ }^{10}$ B. R. Patton and A. Zaringhalam, Phys. Lett. 55A, 95 (1975).

${ }^{11}$ K. Levin and O. T. Valls, Phys. Rev. B 17, 191 (1978); 20, 105 (1979).

${ }^{12}$ We thank K. Levin (private communication) for pointing out the relation of our results to the $\mathrm{PZ}$ formula and its generalizations, and for correcting a factor-of- $(2 l+1)$ error in a previous version of the paper.

${ }^{13}$ B. Bellarbi, A. Benoit, D. Jaccard, J. M. Mignot and H. F. Braun, Phys. Rev. B 30, 1182 (1984).

${ }^{14}$ L. Gao, P. H. Hor, R. L. Meng, Z. L. Du, Z. X. Zhao, and C. W. Chu, Bull. Am. Phys. Soc. 31, 648 (1986).

15 J. O. Willis, J. D. Thompson, Z. Fisk, A. de Visser, J. J. M. Franse, and A. Menovsky, Phys. Rev. B 31, 1654 (1983).

${ }^{16}$ J. A. Olsen, R. A. Fisher, N. E. Phillips, G. R. Stewart, and A. L. Giorgi, Bull. Am. Phys. Soc. 31, 648 (1986). 